\title{
Cortical reactivations during sleep spindles following declarative learning
}

\author{
Aude Jegou $^{\text {a,b,c, } 1}$, Manuel Schabus ${ }^{\mathrm{d}, 1}$, Olivia Gosseries ${ }^{\mathrm{e}, \mathrm{f}}$, Brigitte Dahmen ${ }^{\mathrm{g}}$, \\ Geneviève Albouy $^{\mathrm{h}}$, Martin Desseilles ${ }^{\mathrm{i}}$, Virginie Sterpenich ${ }^{\mathrm{j}}$, Christophe Phillips ${ }^{\mathrm{e}}$, \\ Pierre Maquet ${ }^{\mathrm{e}, \mathrm{f}}$, Christophe Grova ${ }^{\mathrm{a}, \mathrm{b}, \mathrm{k}, 1}$, Thien Thanh Dang-Vu ${ }^{\mathrm{a}, \mathrm{c}, \mathrm{m}, \mathrm{n}, \text {, }}$ \\ ${ }^{\text {a }}$ PERFORM Center, Concordia University, 7200 Sherbrooke St W, H4B 1R6, Montreal, Canada \\ ${ }^{\mathrm{b}}$ Department of Physics, Concordia University, 7141 Sherbrooke St W, H4B 1R6, Montreal, Canada \\ ${ }^{\mathrm{c}}$ Center for Studies in Behavioral Neurobiology (CSBN), Concordia University, 7141 Sherbrooke St W, H4B 1R6, Montreal, Canada \\ d Center for Cognitive Neuroscience (CCNS), Department of Psychology, University of Salzburg, 34 Hellbrunnerstr., 5020, Salzburg, Austria \\ ${ }^{\mathrm{e}}$ GIGA Institute, University of Liège, 1 Avenue de l'Hôpital, 4000, Liege, Belgium \\ ${ }^{\mathrm{f}}$ Department of Neurology, University of Liege, 1 Avenue de l'Hôpital, 4000, Liege, Belgium \\ ${ }^{\mathrm{g}}$ Department of Child and Adolescent Psychiatry, Psychosomatics and Psychotherapy, RWTH Aachen University, Neuenhofer Weg 21, 52074, Aachen, Germany \\ ${ }^{\mathrm{h}}$ Department of Movement Sciences, KU Leuven, 101 Tervuursevest, 3001, Leuven, Belgium \\ ${ }^{\mathrm{i}}$ Department of Psychology, University of Namur, 61 Rue de Bruxelles, 5000, Namur, Belgium \\ ${ }^{\mathrm{j}}$ Department of Neuroscience, University Medical Center, 9 Chemin des Mines, 1202, Geneva, Switzerland \\ ${ }^{\mathrm{k}}$ Department of Biomedical Engineering, McGill University, 3801 University St, H3A 2B4, Montreal, Canada \\ ${ }^{1}$ Department of Neurology and Neurosurgery, McGill University, 3801 University St, H3A 2B4, Montreal, Canada \\ ${ }^{\mathrm{m}}$ Department of Health, Kinesiology and Applied Physiology, Concordia University, 7141 Sherbrooke St W, H4B 1R6, Montreal, Canada \\ ${ }^{\mathrm{n}}$ Centre de Recherche de l'Institut Universitaire de Gériatrie de Montréal (CRIUGM), 4545 Chemin Queen-Mary, M7834, H3W 1W5, Montreal, Canada
}

\section{A R T I C L E I N F O}

\section{Keywords:}

Consolidation

EEG/fMRI

Memory

Oscillations

Replay

Sleep

\begin{abstract}
A B S T R A C T
Increasing evidence suggests that sleep spindles are involved in memory consolidation, but few studies have investigated the effects of learning on brain responses associated with spindles in humans. Here we used simultaneous electroencephalography (EEG) and functional magnetic resonance imaging (fMRI) during sleep to assess haemodynamic brain responses related to spindles after learning. Twenty young healthy participants were scanned with EEG/fMRI during (i) a declarative memory face sequence learning task, (ii) subsequent sleep, and (iii) recall after sleep (learning night). As a control condition an identical EEG/fMRI scanning protocol was performed after participants over-learned the face sequence task to complete mastery (control night). Results demonstrated increased responses in the fusiform gyrus both during encoding before sleep and during successful recall after sleep, in the learning night compared to the control night. During sleep, a larger response in the fusiform gyrus was observed in the presence of fast spindles during the learning as compared to the control night. Our findings support a cortical reactivation during fast spindles of brain regions previously involved in declarative learning and subsequently activated during memory recall, thereby promoting the cortical consolidation of memory traces.
\end{abstract}

\section{Introduction}

There is now a large body of evidence that sleep benefits memory consolidation. Numerous studies have shown that sleep stabilizes memory traces and reduces forgetting of newly encoded information (for review, see (Diekelmann and Born, 2010; Rasch and Born, 2013; Stickgold, 2013)). The underlying neurobiological processes still remain a topic of intense investigation. Beyond the effects of the different stages of sleep on memory consolidation, an increasing emphasis has been placed on specific oscillatory patterns that can be associated with "offline" neuronal plasticity. In particular, sleep spindles have been consistently related to cognitive abilities and memory consolidation (Schabus et al., 2006; Fogel and Smith, 2011; Luthi, 2014).

Spindles are thalamocortical rhythms detected on scalp EEG during

\footnotetext{
* Corresponding author. Center for Studies in Behavioral Neurobiology (CSBN), Concordia University, 7141 Sherbrooke St. West, SP 165.27, Montréal, H4B 1R6, Canada.

E-mail address: tt.dangvu@concordia.ca (T.T. Dang-Vu).

1 These two authors contributed equally to this work.
} 
stages N2-N3 of non-rapid-eye-movement (NREM) sleep as waxing-andwaning oscillations at $11-16 \mathrm{~Hz}$ (Iber et al., 2007). They are generated through the interplay between the thalamic reticular nucleus and thalamocortical neurons, which project their activity to cortical neurons from which EEG spindles can be recorded (Steriade et al., 1993). Spindles are subcategorized into slow and fast spindles, with the slow $(<13 \mathrm{~Hz})$ predominating over frontal EEG derivations and the fast $(>$ or $=13 \mathrm{~Hz})$ over centroparietal derivations (De Gennaro and Ferrara, 2003; Schabus et al., 2007).

A role for spindles in sleep-related memory consolidation has been suggested by studies showing that learning increases spindle activity during sleep. For example, in humans, intense verbal learning of word pairs increased spindle activity during subsequent sleep (Gais et al., 2002), which was associated with a better memory recall after sleep (Schabus et al., 2004). In rats, an increase in spindle activity was observed after an odor-reward association task (Eschenko et al., 2006). In addition, several studies showed that experimental manipulations enhancing spindle activity - for instance with transcranial alternating current stimulation (Lustenberger et al., 2016; Ketz et al., 2018) or transcranial direct current stimulation (Marshall et al., 2004; Koo et al., 2018) - also increased memory recall after sleep. These studies add more causal evidence that sleep spindles (as well as slow oscillations) play a role in the offline consolidation of memory traces.

To better understand the neural processes underlying the generation and properties of sleep spindles, brain responses during spindles were investigated using simultaneous EEG/fMRI, showing increased responses in the thalamus and several cortical areas in line with spindle generation mechanisms (Schabus et al., 2007; Andrade et al., 2011; Caporro et al., 2012). When contrasting slow and fast spindles, only fast spindles were found associated with responses in the hippocampus and medial prefrontal cortex (Schabus et al., 2007). In addition, functional connectivity between the hippocampus and neocortex was enhanced during fast spindles (Andrade et al., 2011). These findings are in line with the increased coupling between spindles and hippocampal ripples found after declarative learning both in humans and animals (Sirota et al., 2003; Molle et al., 2009). Such interplay might promote the consolidation during NREM sleep of declarative memories, through the transfer of reactivated information from hippocampal to neocortical sites during fast spindles (Rasch and Born, 2013).

There is yet little evidence in humans to support an actual reactivation of learning-related areas during sleep or sleep spindles, with some notable exceptions (Peigneux et al., 2004; Rasch et al., 2007; Fogel et al., 2017). To our knowledge, only one neuroimaging study has assessed brain responses to spindles in relation to learnt declarative material. In that study on 9 participants after partial sleep deprivation, learning prior to sleep was associated with larger brain responses in the hippocampus and task-related cortical regions during fast spindles (Bergmann et al., 2012). These responses were observed in association with fast spindle amplitude and correlated with learning performance before sleep but not with memory change over sleep. Therefore it was unclear whether these spindle-associated brain activations actually promoted the overnight consolidation of memory traces or rather indicated a relation of spindles with general memory aptitude or "intelligence" (Wislowska et al., 2016). In addition, sleep restriction prior to learning might have affected the observed results.

Our objective was to further investigate the neural mechanisms of spindle-related memory consolidation using EEG-fMRI during sleep after declarative learning in a sample of non-sleep deprived individuals. Participants were scanned using simultaneous EEG/fMRI during 2 nights separated by a 1-week interval, with the first night as the learning night and the other as the control night (Fig. 1). In both nights, participants were scanned 1) while encoding a face sequence learning task (Kumaran and Maguire, 2006), 2) during subsequent sleep, 3) and during a recall after awakening. During the 1 -week interval, participants were trained daily on the identical learning task but outside the scanner to achieve over-learning and complete mastery of the task. The repeated training allowed participants to reach by the time of the control night a level of task exposure at which they had no relevant information left from the task to learn or consolidate, while being exposed to the exact same stimulus material as during the learning night.

Our main analysis assessed the changes in brain responses to spindles between the learning and control nights, with a focus on the differential response between fast and slow spindles as only fast spindles have previously been shown to recruit hippocampo-neocortical networks (Schabus et al., 2007). We hypothesized that fast spindles compared to slow spindles would be associated with increased responses in brain regions involved in prior encoding of the task before sleep and in successful recall of the task after sleep. In addition, we sought to test whether these spindle-related changes in brain responses would be associated with the overnight consolidation of declarative memory. We hypothesized that

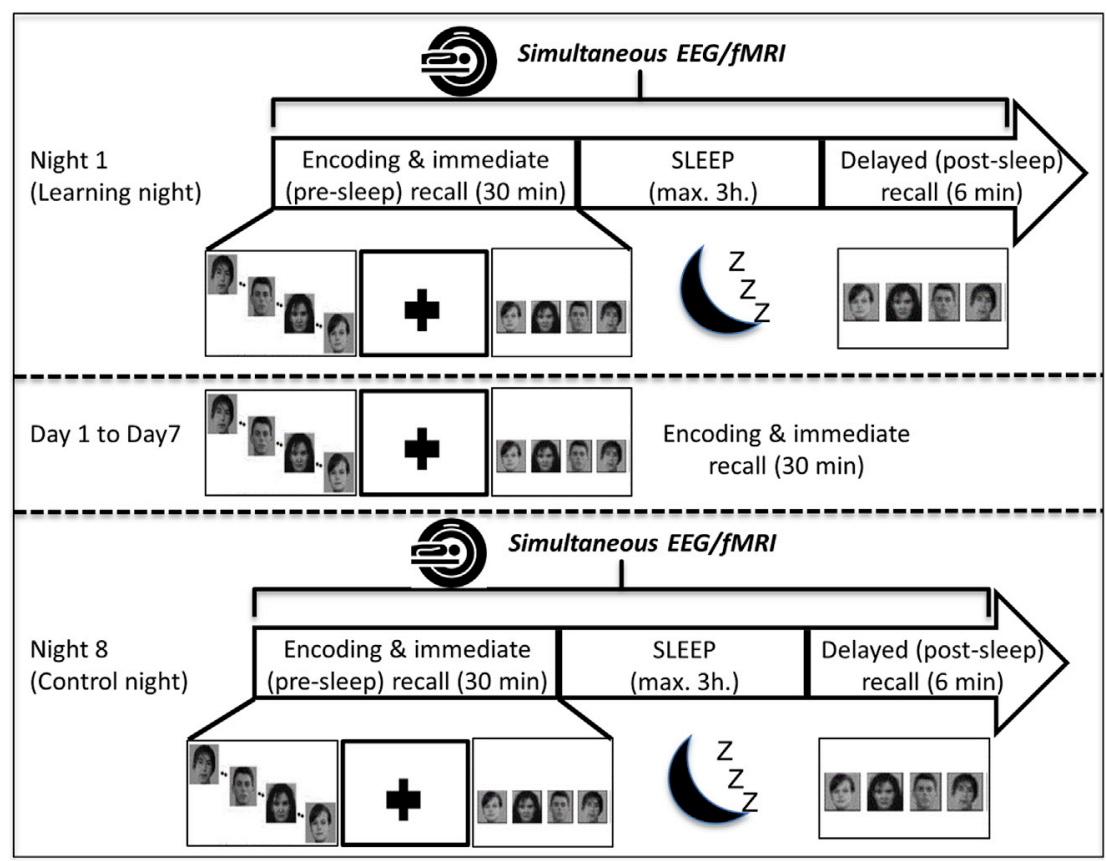

Fig. 1. Experimental protocol.

All participants completed two nights of EEG/fMRI recordings during which they were exposed to a face sequence learning task with immediate (pre-sleep) recall, followed by a sleep period in the scanner and then a delayed (post-sleep) recall upon awakening. The two nights were separated by a 1-week interval during which participants returned to the lab daily for re-exposure to the same learning task. The first EEG/fMRI night was thus used as a learning condition, while the second EEG-fMRI night was used as the control condition given the exposure to the same material without additional learning. 
overnight memory change would be related to spindle-associated reactivations, thereby linking brain activity during spindles with the offline consolidation of memory traces.

\section{Materials and methods}

\subsection{Participants}

20 young, healthy, non-sleep deprived, right-handed participants (10 females, age range 19-27) gave their written informed consent and received financial compensation for their participation in this study, which was approved by the Ethics Committee of the Faculty of Medicine of the University of Liège, Belgium. 14 of them were included in the final analyses, as 6 participants had to be excluded due to technical issues or inability to reach stable sleep in the scanner in one of the two nights of the protocol. Participants were free of any history of medical, traumatic, psychiatric or sleep disorder, as assessed by a semi-structured interview. All participants were non-smokers, moderate caffeine and alcohol consumers. None were on any medication. They were not sleep-deprived, as they were instructed to follow a regular sleep-wake schedule assessed by wrist actigraphy (Actiwatch, Cambridge Neuroscience, UK) and sleep diaries starting 4 days prior to first visit at the laboratory and continuing throughout the whole duration of protocol. None had worked on night shifts during the last year or travelled through more than one time zone during the last 2 months. Participants were requested to refrain from all caffeine and alcohol-containing beverages and intense physical activity for 3 days prior to the first visit at the laboratory and throughout the whole study.

\subsection{Experimental design}

A schematic of the protocol is illustrated in Fig. 1. Participants were scheduled for simultaneous EEG-fMRI recordings in the laboratory during 2 visits at a 1-week interval. At each of these visits, they reported to the MRI laboratory at the Cyclotron Research Centre (University of Liège, Belgium) at 8pm and both visits followed the same sequence of events and scanning sessions. First, actigraphy and sleep diaries were checked for compliance with the regular sleep schedule and the absence of sleep deprivation. Then the MRI-compatible EEG cap was set up and participants were installed in the MRI scanner in order to start the simultaneous EEG-fMRI acquisitions. In both visits, EEG-fMRI acquisitions included three consecutive sessions, taking place during the first half of the night: 1) a learning session during which participants performed a declarative task, which consisted of remembering the order of neutral faces presented in pairs of overlapping sequences (Kumaran and Maguire, 2006), with an immediate recall to assess pre-sleep learning performance; 2) a sleep session during which participants slept in the scanner (for a maximum of $3 \mathrm{~h}$ ); 3) a delayed recall session to evaluate post-sleep memory retention. Between the two visits, participants were instructed to come to the lab every day at the same time in the late afternoon for a short learning session outside the scanner. During these daily sessions, participants performed the same declarative learning task with the same sequences of faces as during the initial visit. This protocol was designed to allow participants to achieve complete learning of the task before their second EEG-fMRI visit. Therefore the first overnight visit served as the learning night, while the second overnight visit - one week later - was used as a control night given that participants had no new relevant material to learn while being exposed to the same tasks and stimuli during the night.

\subsection{Declarative learning task}

The task was based on a declarative learning paradigm that was previously used in fMRI (Kumaran and Maguire, 2006) (Fig. 1). During the encoding session, participants were instructed to remember sequences of faces that were presented on a screen while lying on the MR scanner table in the evening of the first night in the laboratory (see Supporting Information, SI, for details). An immediate recall followed the presentation of each sequence of faces, and the average performance (pre-sleep) was calculated across sequences for a maximal score of 18. Participants then slept in the scanner, and after the sleep session, they had a break of $30 \mathrm{~min}$ outside the scanner during which they were exposed to bright light. They were then installed again on the MRI scanner table for a delayed recall session (i.e., post-sleep), and the post-sleep recall performance was likewise computed. The difference in recall performance between the delayed (post-sleep) and immediate (pre-sleep) recall was calculated to provide a score of overnight change in recall performance, reflecting memory retention after sleep. From day 1 to day 7 following the first night at the laboratory, participants came back to the laboratory every day during the afternoon to perform the same encoding session (and immediate recall) with the same face sequences as during the first night but on a computer outside the MRI scanner. During night 8 (control night), the same series of tasks consisting of the same face sequences were used in the MRI scanner as during night 1 (learning night) and served as a control condition.

\subsection{EEG acquisition and analysis}

During both learning and control nights, EEG was recorded simultaneously with fMRI using two MR-compatible 32-channel amplifiers (BrainAmp MR plus, Brain Products GmbH, Gilching, Germany) and a MR-compatible EEG cap (BrainCap MR, Falk Minow Services, Herrsching Breitbrunn, Germany) including 64 ring-type electrodes (see SI for details). The recording setup was similar to our previous EEG-fMRI studies (Schabus et al., 2007; Dang-Vu et al., 2008; Dang-Vu et al., 2011). For analysis, EEG were first cleaned for scanner gradient artefacts and ballistocardiogram artefacts (Allen et al., 2000; Leclercq et al., 2009; Leclercq et al., 2011). Sleep staging followed standard criteria (Iber et al., 2007) and identified periods of stages N2-N3 sleep, free of any artefact, arousal or shifts in vigilance states, during which the EEG and fMRI data were analyzed. In these epochs, slow and fast spindles were automatically detected on $\mathrm{Fz}, \mathrm{Cz}$ and $\mathrm{Pz}$, using an algorithm adapted from Mölle and colleagues (Molle et al., 2002) and used in our previous studies (Schabus et al., 2007; Dang-Vu et al., 2011). In the same epochs, slow wave activity was also computed as the square root of the energy of the signal in the 0-5-4 Hz frequency band averaged within temporal windows of $2.46 \mathrm{~s}$, corresponding to each repetition time of the fMRI sequence.

\section{5. fMRI data acquisition and analysis}

Multislice T2*-weighted fMRI images were obtained using a 3 T MR scanner (Allegra, Siemens, Germany), with a gradient echo-planar sequence using axial slice orientation and reduced slew rate for efficient gradient artefact rejection and reduced acoustic noise (32 slices; voxel size: $3.4 \times 3.4 \times 3 \mathrm{~mm}^{3}$; matrix size $=64 \times 64 \times 32$; $\mathrm{TR}=2460 \mathrm{~ms}$; $\mathrm{TE}=40 \mathrm{~ms}$; flip angle $=90^{\circ}$; delay $=0$ ). Slices were acquired sequentially in an ascending order. Subjects were scanned during encoding, immediate (pre-sleep) recall, sleep and delayed (post-sleep) recall sessions of nights 1 and 8, while fMRI and EEG data were acquired continuously. During the sleep session, they were asked to relax and try to sleep in the scanner. The sleep session was stopped when the participant indicated by button press the wish to leave the scanner, or for a maximum of 4000 scans (about $164 \mathrm{~min}$ ). A structural T1-weigthed 3D MP-RAGE sequence $(\mathrm{TR}=1960 \mathrm{~ms}, \mathrm{TE}=4.43 \mathrm{~ms}$, inversion time $=1100 \mathrm{~ms}$, FOV $=230 \times 173 \mathrm{~mm}^{2}$, matrix size $=256 \times 192 \times 176$, voxel size $=0.9 \times 0.9 \times 0.9 \mathrm{~mm}^{3}$ ) was also acquired in all subjects for display of results.

Functional volumes were analyzed using Statistical Parametric Mapping 8 (SPM8 - http://www.fil.ion.ucl.ac.uk/spm/software/spm8/) implemented in MATLAB (version 2010b, MathWorks Inc, Natick). Functional MRI time series were corrected for slice timing, rigid head motion, spatially normalized (voxel size $=2 \times 2 \times 2 \mathrm{~mm}^{3}$ resampled using 
spline interpolation) to an echo planar imaging template conforming to the Montreal Neurological Institute (MNI) space, and spatially smoothed with a Gaussian Kernel of $8 \mathrm{~mm}$ full width at half maximum (FWHM). The analysis of fMRI data, based on a mixed effects model, was conducted in two successive steps, accounting respectively for intra-individual (fixed effects, first-level) and inter-individual (random effects, secondlevel) variance. The analysis aimed at: 1) characterizing brain responses associated with task encoding before sleep and recall after sleep; 2) characterizing the effect of learning on brain responses associated with sleep spindles. For each participant, brain responses were modelled at each voxel using a general linear model.

In order to assess the fMRI responses to task encoding and recall, four trial types were modelled (visualisation of face sequences, fixation cross, pre-sleep recall, post-sleep recall) across the encoding and recall sessions of learning and control nights for each subject, convolved with the haemodynamic response function (HRF), and used as regressors of interest in the individual design matrix. The contrasts of interest were those related to task encoding (visualisation of face sequences) and differences in task recall after sleep (contrasting post-sleep versus pre-sleep recalls), during the learning night as compared to the control night.

For the assessment of spindle responses, the vectors including the onsets of detected fast and slow spindles were first convolved with the three canonical basis functions (HRF, its derivative and dispersion as suggested in (Friston et al., 1998)) across the sleep sessions of learning and control nights for each subject, and then used as regressors of interest in another individual design matrix. In the latter, two additional regressors, convolved with the HRF, were included as confounding variables of no interest: one containing slow wave activity as we aimed at identifying responses to spindles over and above those to slow waves, and another including R-R intervals derived from ECG to take into account the artefacts related to the cardiac cycle. Note that the regressor containing slow wave activity was removed in an additional analysis, exploring the responses to spindles regardless of the presence of concurrent slow waves. The sleep contrasts were focused on responses to fast spindles when compared to slow spindles, as the differential response to fast versus slow spindles was found to recruit both hippocampal and cortical responses in our previous studies (Schabus et al., 2007). Contrasting these two spindle subtypes also allowed a direct comparison of spindle responses between learning night and control night, by subtracting the potentially confounding effect of baseline activity from the comparison between the 2 nights. In order to estimate the effects of learning on spindle responses, the main contrast for the sleep session thus consisted of responses to fast spindles minus slow spindles for the learning night compared to fast minus slow spindles for the control night in each subject.

For all analyses, movement parameters estimated during realignment (translations and rotations around $x, y$, and $z$ axes) and a constant vector were included in the matrix as confounding regressors of no interest. All analyses also included a high-pass filtering using a cut-off period of $128 \mathrm{~s}$ to remove low frequency drifts from the fMRI time series. Serial correlations in fMRI signal were estimated with an autoregressive (order 1) plus white noise model using a restricted maximum likelihood (ReML) algorithm to estimate the noise model.

All individual contrast images were then entered in second-level analyses: one-sample t-tests for the encoding and recall contrasts and ANOVAs for the spindle contrasts. For the sleep sessions, the canonical basis set (3 levels corresponding to the HRF mode, its derivative and dispersion) was entered as factor to estimate changes in fast versus slow spindles across subjects and across the three basis functions. In order to evaluate the effects of sleep-related memory consolidation in these different contrasts, task performance results were entered as covariates in the second-level analyses. Task performance results consisted in differences between learning and control nights in delayed (post-sleep) minus immediate (pre-sleep) recall performance values. For completeness, we also used differences between learning and control nights in pre-sleep recall performance for supplementary analyses evaluating the effects of pre-sleep learning performance on brain responses to encoding and spindles. The error covariance was not assumed independent between regressors and a correction for non-sphericity was applied. The resulting set of voxel values constituted maps of $t$ [SPM(t)] or $F$ statistics [SPM(F)]. To correct for multiple comparisons, statistical inferences were reported in regions of interest (ROIs) previously identified in neuroimaging studies of face learning (Clark et al., 1998; Vuilleumier P et al., 2001; Pourtois G et al., 2005; Kumaran and Maguire, 2006) and sleep spindles after learning (Bergmann et al., 2012; Fogel et al., 2017) using spherical volumes $\left(10 \mathrm{~mm}\right.$ radius sphere, i.e. $\sim 4000 \mathrm{~mm}^{3}$; small volume correction [SVC]), and a threshold of $p<0.05$ corrected for multiple comparisons at the voxel level. For the learning and recall contrasts, these ROIs included the fusiform gyrus $(x=44, y=-54, z=-20 ; x=-36, y=-74$, $\mathrm{z}=-19 ; \mathrm{x}=-45, \mathrm{y}=-51, \mathrm{z}=-27)$, inferior frontal gyrus $(\mathrm{x}=-45$, $\mathrm{y}=21, \mathrm{z}=24)$ and hippocampus $(\mathrm{x}=21, \mathrm{y}=-33, \mathrm{z}=-12)$. For the spindle contrasts, ROIs included the fusiform gyrus $(x=44, y=-66$, $\mathrm{z}=-20)$, thalamus $(\mathrm{x}=2, \mathrm{y}=-18, \mathrm{z}=17)$ and hippocampus $(\mathrm{x}=21$, $\mathrm{y}=-33, \mathrm{z}=-12$ ). An additional correction, using Bonferroni, was applied to take into account the number of ROIs investigated for the learning and recall contrasts, and the number of ROI(s) potentially reactivated from learning to spindles.

\section{Results}

Out of 20 participants who entered the protocol, 4 were not able to reach stable sleep in one of the two EEG/fMRI sessions and data from 2 participants could not be used for technical reasons (with MRI scanner or EEG recording). Thus data from 14 subjects were used for subsequent data analysis. For the sleep EEG-fMRI sessions, only epochs of stage N2 and N3 were considered for analyses, as the study focused on spindles. There was no difference in the duration of $\mathrm{N} 2$ or N3 between the 2 nights (paired t-tests, $\mathrm{p}>0.05$ ), although participants spent more time in the scanner and slept longer overall during the control night as to be expected with the habituation to the scanner environment. Fast and slow spindles were detected offline on these N2-N3 epochs, and no significant difference was found in the number or density (nb of spindles/minute) of fast and slow spindles between nights (paired t-tests, $\mathrm{p}>0.05$ ) (Table 1).

At the behavioural level, subjects showed an immediate (pre-sleep) recall performance score of $15.23 \pm 1.42$ (mean \pm SD) and a delayed (post-sleep) recall score of $15.1 \pm 1.8$ during the learning night. After one week of repeated practice, during the control night, participants displayed a pre-sleep recall performance of $17.79 \pm 0.24$ and a post-sleep recall score of $17.76 \pm 0.34$, thus reaching or closely approaching the maximum score of 18 (all scores during the control night ranged from 17

Table 1

Demographic and sleep characteristics of participants.

\begin{tabular}{llll}
\hline & Learning Night & Control Night & p-value \\
\hline Sample size & $14(6 \mathrm{~F}$ and 8M) & Same & N/A \\
Age (years) & $22 \pm 1.1$ & $22 \pm 1.1$ & N/A \\
Time in scanner (min) & $124.94( \pm 35.8)$ & $141.18( \pm 39.38)$ & 0.02 \\
Sleep duration (min) & $64.54( \pm 43.9)$ & $90.53( \pm 38.95)$ & 0.02 \\
Stage N1 duration (min) & $5.35( \pm 8.45)$ & $14.23( \pm 18.19)$ & 0.05 \\
Stage N2 duration (min) & $40.38( \pm 37.8)$ & $55.89( \pm 32.61)$ & 0.09 \\
Stage N3 duration (min) & $18.81( \pm 25.4)$ & $20.41( \pm 26.56)$ & 0.35 \\
Number of fast spindles & $119.14( \pm 147.4)$ & $120.43( \pm 119.66)$ & 0.49 \\
Number of slow spindles & $21.36( \pm 27.4)$ & $22.86( \pm 34.18)$ & 0.43 \\
Fast spindle density (nb/min) & $1.44( \pm 0.96)$ & $1.31( \pm 0.99)$ & 0.31 \\
Slow spindle density (nb/min) & $0.28( \pm 0.21)$ & $0.26( \pm 0.33)$ & 0.36 \\
Fast spindle duration (sec) & $0.68( \pm 0.14)$ & $0.65( \pm 0.08)$ & 0.53 \\
Slow spindle duration (sec) & $0.58( \pm 0.19)$ & $0.51( \pm 0.16)$ & 0.31 \\
\hline
\end{tabular}

This table lists the demographic information of the participants as well as the characteristics of their sleep sessions during the EEG-fMRI recordings for both the learning and the control nights. Values correspond to means ( \pm standard deviations). Differences between nights were tested by paired t-tests, and the $\mathrm{p}$ values for these comparisons are displayed in the right column. $\mathrm{F}=$ female; $\mathrm{M}=$ male; $\mathrm{N} / \mathrm{A}=$ non applicable; $\min =$ minutes; $\mathrm{nb}=$ number; $\mathrm{sec}=$ seconds. 
to 18), as expected from the over-learning paradigm (Fig. 2).

To assess the effects of learning, we compared brain responses during the encoding of face sequences between the learning and control night. There was a significantly larger response in the fusiform gyrus bilaterally (extending to the vicinity of the parahippocampal gyrus) during pre-sleep task encoding of the learning night compared to the control night (Table 2, Fig. 3A). In addition, responses in the fusiform gyrus and inferior frontal gyrus during encoding were positively correlated with pre-sleep recall performance (Table S1 in Supporting Information, SI). To assess the effects of task recall after sleep, we then compared brain responses between post-sleep and pre-sleep recall, during the learning night as compared to the control night, including the corresponding performance change as a covariate. We found a significantly larger response in the fusiform gyrus, extending to the parahippocampal gyrus, during the post-sleep versus pre-sleep recall of the learning compared to control night, which was positively correlated with the corresponding change in recall performance (Table 2, Fig. 3B). The result indicates that higher fusiform gyrus activation during recall after sleep was associated with better retrieval performance.

Our main analysis evaluated changes in brain responses during spindles of N2-N3 sleep of the learning versus control night, comparing fast to slow spindles as previous EEG/fMRI studies linked fast spindles to regions involved in declarative learning (Schabus et al., 2007; Bergmann et al., 2012). Fast spindles compared to slow spindles were associated with a significantly larger response in the right fusiform gyrus during the learning versus control night (Table 3, Fig. 4A). In addition, we found a trend for a larger response in the left thalamus using the same contrast (Fig. 4A). During fast (versus slow) spindles of the learning compared to control night, responses in the thalamus were positively correlated with pre-sleep learning performance (Table S2 in SI).

When recall performance change (post-minus pre-sleep) was included as a covariate, we found a trend for a larger response in the right hippocampus in association with fast spindles (minus slow spindles) (learning-control night), which was positively correlated with the corresponding change in memory recall performance. This result suggests that better retrieval (i.e., less forgetting) after sleep could be associated with larger fast spindle-related hippocampal responses during sleep (Table 3, Fig. 4B). There was no significant response with fast compared to slow spindles between nights when slow wave activity was removed from the covariates.

\section{Discussion}

Using simultaneous EEG/fMRI, we assessed the neuronal responses to fast spindles during sleep following learning of a new declarative memory task consisting of face sequences. A control condition consisted of the identical learning task, which was learnt daily across a week to reach a ceiling effect in performance. This re-exposure was aimed at providing a control condition in which participants would be exposed to the exact same set of stimuli before sleep on the control night of the study protocol, yet with no relevant information left to learn. As expected, participants reached by the second EEG/fMRI session a score that was at (or very close to) the maximum (Fig. 2), in line with our assumption that task learning was fully (or almost) completed before the control night.

Our first finding was the involvement of the fusiform gyrus not only in encoding the face sequence task, but also in the successful recall of this
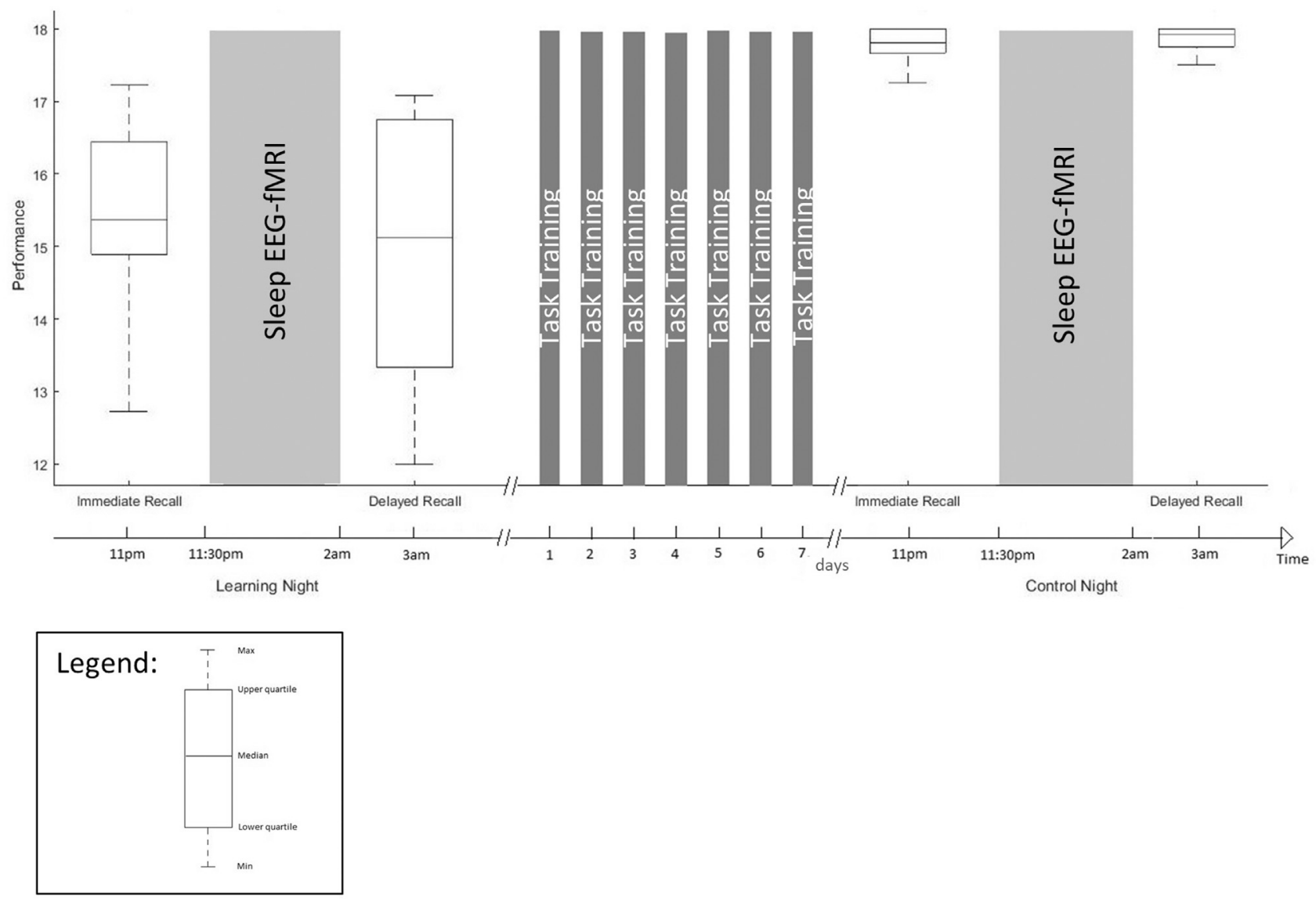

Fig. 2. Behavioural performances.

This box plot shows the recall performance scores (median, upper/lower quartiles) before (immediate) and after (delayed) sleep, during the learning night and the control night. Note that scores reached or approached the maximum recall performance of 18 during the control night, demonstrating full mastery of the task as expected from the protocol. 
Table 2

Brain responses to task learning and task recall.

\begin{tabular}{|c|c|c|c|c|c|c|c|c|c|c|c|}
\hline \multirow[t]{2}{*}{ Region } & \multirow[t]{2}{*}{ Side } & \multicolumn{5}{|c|}{ Encoding (learning - control night) } & \multicolumn{5}{|c|}{$\begin{array}{l}\text { Recall (learning - control night), modulated by Recall } \\
\text { Performance (post-sleep - pre-sleep) }\end{array}$} \\
\hline & & $x$ & $y$ & $z$ & $Z$ score & $P_{S V C}$ & $x$ & $y$ & $z$ & $Z$ score & $P_{S V C}$ \\
\hline \multirow{4}{*}{$\begin{array}{l}\text { Fusiform gyrus (Clark et al., 1998; Vuilleumier et al., 2001; } \\
\text { Pourtois et al., 2005) }\end{array}$} & $\mathrm{R}$ & 49 & -50 & -15 & 4.19 & $<0.01 *$ & \multirow[t]{3}{*}{46} & \multirow[t]{3}{*}{-64} & \multirow[t]{3}{*}{-11} & \multirow[t]{3}{*}{3.15} & \multirow[t]{3}{*}{0.04} \\
\hline & $\mathrm{R}$ & 39 & -47 & -19 & 3.27 & 0.03 & & & & & \\
\hline & $\mathrm{R}$ & 42 & -50 & -15 & 3.31 & 0.03 & & & & & \\
\hline & $\mathrm{L}$ & -40 & -74 & -11 & 3.39 & $<0.01^{*}$ & -40 & -47 & -19 & 3.37 & $0.01 *$ \\
\hline Inferior Frontal gyrus (Kumaran and Maguire, 2006) & $\mathrm{L}$ & -50 & 15 & 24 & 3.40 & 0.03 & & & & & \\
\hline
\end{tabular}

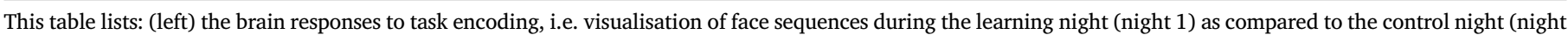

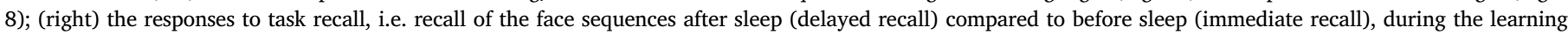

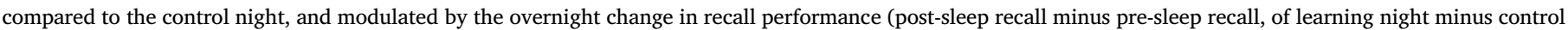

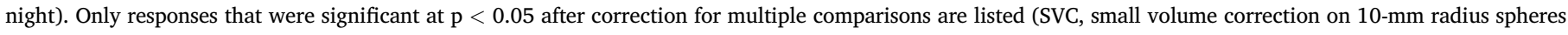

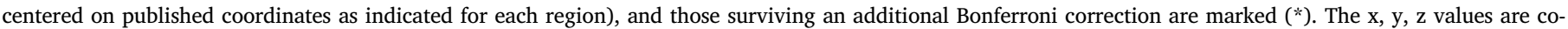
ordinates (in mm) in the standard Montreal Neurological Institute (MNI) space.

\section{A. Brain responses to task encoding (before sleep, learning - control night)}
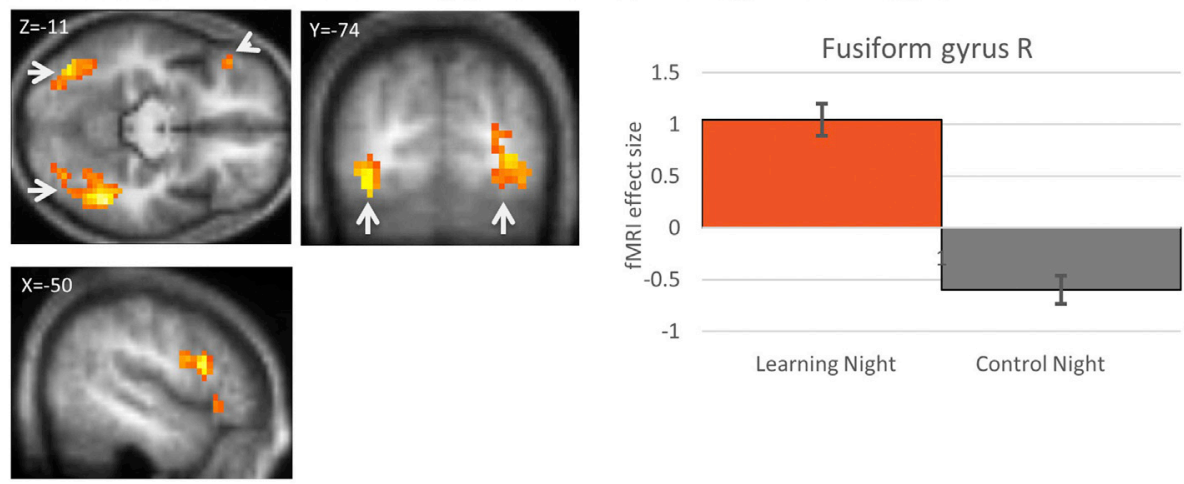

B. Brain responses changes to task recall $x$ overnight memory change (after - before sleep, learning - control night)
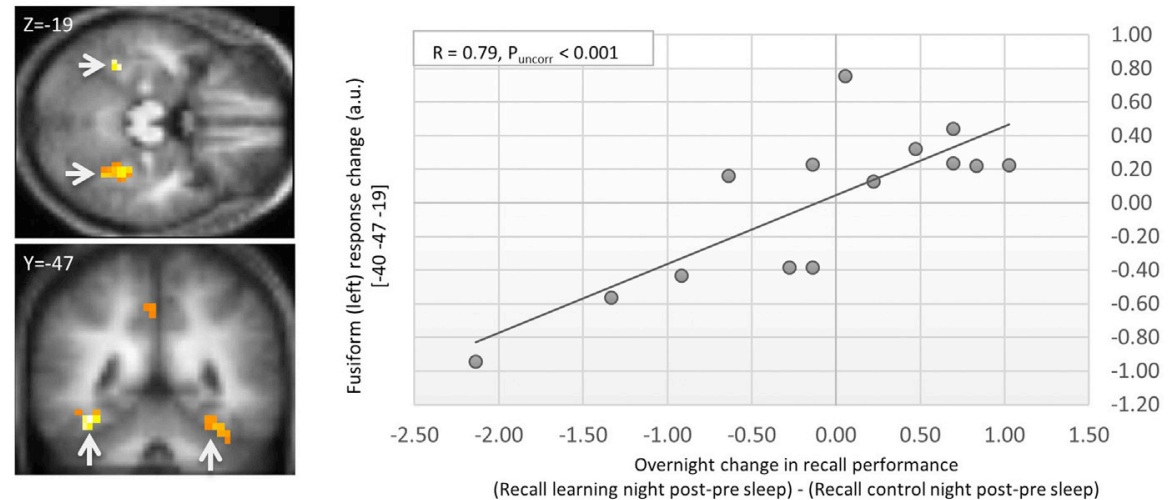

(Recall learning night post-pre sleep) - (Recall control night post-pre sleep)
Fig. 3. Brain responses to task encoding and task recall.

(A) Brain responses to task encoding (visualisation of face sequences) during the learning night as compared to the control night. Significant responses were located in the fusiform gyrus bilaterally (white arrows) ( $<0.05$ after correction for multiple comparisons, SVC). Results are displayed at an uncorrected $\mathrm{p}<0.02$, on the mean structural MRI image, and shown on transverse (left), coronal (middle) and parasagittal (right) sections ( $x, y$ and $z$ levels indicated for each section). Bar graphs represent the contrast between average responses in the right fusiform gyrus during task encoding in the learning vs control night. (B) Brain responses to task recall following sleep (delayed recall) as compared to before sleep (immediate recall) during the learning compared to control night, and modulated by the overnight change in recall performance (post-sleep minus pre-sleep recall, of learning night minus control night).

(Left panels) Significant responses were located in the fusiform gyrus bilaterally (white arrows) $\left(\mathrm{p}_{\mathrm{svc}}<0.05\right.$ corrected for multiple comparisons). Results are displayed at an uncorrected $\mathrm{p}<0.02$, on the mean structural MRI image, and shown on transverse (top) and coronal (bottom) sections ( $y$ and $z$ levels indicated for each section).

(Right panel) Scatter plot illustrating the effects reported in the left fusiform gyrus $(x=-40, y=-47$, $\mathrm{z}=-19$ ) for this contrast: response changes (in arbitrary units, a.u.) in this region during post-sleep (versus pre-sleep) recall of the learning (vs control) night were positively correlated with the overnight change in recall performance. The coefficient $\mathrm{R}$ for this correlation was 0.79 , for an uncorrected $p$ value ( $\mathrm{p}_{\text {uncorr }}$ ) $<0.001$, corresponding to a corrected $\mathrm{p}_{\text {svc }}=0.01$ (see Table 2, right column). task after sleep. This region of the fusiform gyrus encompassed the fusiform face area, known to be selectively involved in face perception and identification (Kanwisher et al., 1997; Grill-Spector et al., 2004). Interestingly, our findings also showed that the stronger this region was activated during post-sleep recall the better the memory performance was after sleep (Fig. 3). Our main results then showed increased responses in the fusiform gyrus with fast spindles (versus slow spindles) during the learning as compared to the control night (Fig. 4A). Together these results indicate that overlapping cortical regions were activated (i) when subjects learnt a new declarative task consisting of face sequences before sleep, (ii) during subsequent sleep when fast sleep spindles were present, and (iii) when participants successfully recalled these face sequences after sleep (Fig. 5). These repeated cortical activations in similar regions suggest the presence of "offline" replay mechanisms during fast spindles, which translated into stronger fusiform response during fast spindles after learning. Note that all these effects were contrasted against a control night during which subjects followed an identical study protocol and were exposed to the same stimuli but with no new learning for the participants, which supports the specificity of these findings in relation to the learning paradigm. Thus, our findings provide evidence for memory reactivation during fast spindles in cortical regions that were previously involved in learning the task and subsequently involved in successful recall, in line with a role for fast spindles in the offline consolidation of declarative memory traces. Given that spindle activity has shown to be modulated by the phase of the slow oscillation (Molle et al., 2002; Molle et al., 2011), spindle-related reactivation could have 
Table 3

Brain responses to sleep spindles after learning.

\begin{tabular}{|c|c|c|c|c|c|c|c|c|c|c|c|}
\hline \multirow[t]{2}{*}{ Region } & \multirow[t]{2}{*}{ Side } & \multicolumn{5}{|c|}{ Sleep Spindles (fast - slow, learning night - control night) } & \multicolumn{5}{|c|}{$\begin{array}{l}\text { Sleep Spindles (fast - slow, learning night - control night) modulated } \\
\text { by Recall Performance (post-sleep - pre-sleep recall) }\end{array}$} \\
\hline & & $x$ & $y$ & $z$ & $Z$ score & $P_{S V C}$ & $x$ & $y$ & $z$ & $Z$ score & $P_{S V C}$ \\
\hline $\begin{array}{l}\text { Fusiform gyrus (Bergmann } \\
\text { et al., 2012) }\end{array}$ & $\mathrm{R}$ & 42 & -64 & -11 & 3.02 & $0.04 *$ & & & & & \\
\hline Thalamus (Fogel et al., 2017) & $\mathrm{L}$ & -6 & -19 & 20 & 3.08 & 0.06 & & & & & \\
\hline $\begin{array}{l}\text { Hippocampus (Kumaran and } \\
\text { Maguire, 2006) }\end{array}$ & $\mathrm{R}$ & & & & & & 22 & -26 & -7 & 2.87 & 0.06 \\
\hline
\end{tabular}

This table lists: (left) the brain responses to fast spindles as compared to slow spindles, during the learning night (night 1) as compared to the control night (night 8); (right) the responses to fast versus slow spindles during learning compared to control night, and modulated by the overnight change in recall performance (post-sleep recall minus pre-sleep recall, of learning night minus control night). Responses were corrected for multiple comparisons on 10-mm radius spheres (SVC) centered on published coordinates as indicated for each region. Only responses that remained significant $(p<0.05)$ or were marginally significant $(p<0.1)$ after correction are listed, and those surviving an additional Bonferroni correction are marked $(*)$. The $\mathrm{x}, \mathrm{y}, \mathrm{z}$ values are coordinates in the MNI space.

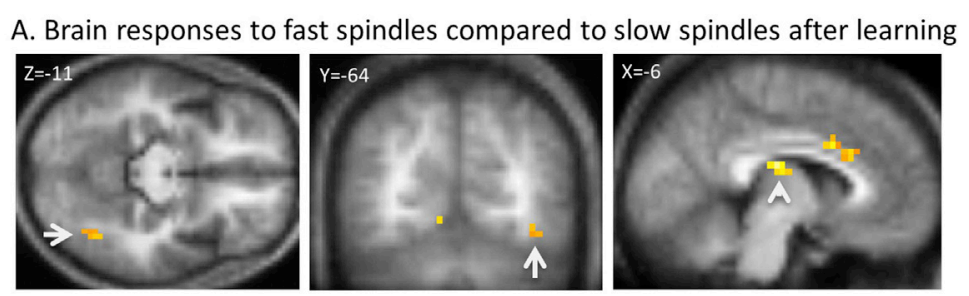

B. Brain responses to fast spindles compared to slow spindles, modulated by overnight change in recall performance
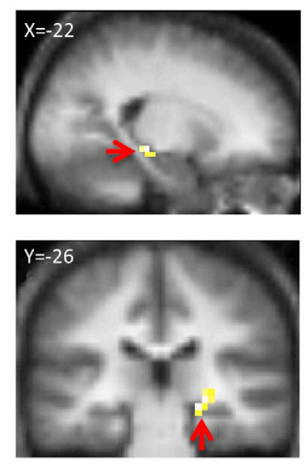

Fig. 4. Brain responses to sleep spindles after learning.

(A) Brain responses to fast (versus slow) spindles during the learning compared to the control night:

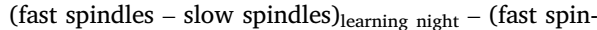
dles - slow spindles) control night. A larger response was found in the right fusiform gyrus (white arrow; $\mathrm{p}_{\mathrm{svc}}<0.05$ corrected for multiple comparisons), with a trend for a larger response in the thalamus (arrow head, $\mathrm{psvc}_{\mathrm{svc}}=0.06$ ). Display at $\mathrm{p}<0.02$ uncorrected, on the mean structural MRI.

(B) Brain responses to fast (versus slow) spindles during the learning compared to the control night and modulated by overnight change in recall performance (post-sleep minus pre-sleep recall of learning minus control night).

(Left panels) A trend for a larger response was observed in the right hippocampus (red arrows) ( $\mathrm{p}_{\mathrm{svc}}=0.06$ corrected). Results are displayed at an uncorrected $\mathrm{p}<0.02$, on the mean structural MRI image, and shown on parasagittal (top) and coronal (bottom) sections ( $x$ and $y$ levels indicated for each section).

(Right panel) Scatter plot illustrating the effects reported in the right hippocampus $(x=22, y=-26$, $\mathrm{z}=-7$ ) for this contrast: response changes (in arbitrary units, a.u.) in this region during fast (versus slow) spindles of the learning (vs control) night were positively correlated with the overnight change in recall performance. $\mathrm{R}$ coefficient for this correlation was 0.71 , for $\mathrm{p}_{\text {uncorr }}<0.01$, corresponding to corrected $\mathrm{p}_{\mathrm{svC}}=0.06$ (Table 3 , right column). been likewise dependent on ongoing slow oscillations. However, the reported effects were found after slow wave activity was included as a covariate. This suggests that brain reactivations with fast spindles were observed above and beyond the effects of slow waves. Accordingly, no significant responses were found with fast spindles when the slow wave activity regressor was removed from the analysis. Our findings therefore suggest that sleep spindles not phase-locked to slow oscillations might also contribute to the reorganization of memory traces after learning.

It has been hypothesized that the role of spindles in declarative memory consolidation involves the transfer of information from hippocampal to neocortical regions (Rasch and Born, 2013). In line with this model, we found a nearly significant correlation ( $\mathrm{p}=0.06$ corrected for multiple comparisons) between hippocampal activity during fast spindles of the learning night (as compared to the control night) and the overnight change in recall performance (Fig. 4B). In other words, over and above a general reactivation of the fusiform gyrus that was consistently observed after learning during fast spindles, we observed a trend for a more specific increase in the hippocampus during fast spindles, which was found only for those individuals who improved (or forgot less) overnight.
Together these findings point to the importance of the hippocampus for an efficient transfer of information to long-term storage during the offline memory consolidation. According to this interpretation, a reactivation of the hippocampus during fast spindles facilitates a more efficient redistribution of memory traces from the hippocampus to long-term storage in the neocortex, which in turn improves memory recall and the stabilization of memory representations.

A previous EEG/fMRI study showed a positive correlation between fast spindle-related responses in the hippocampus and immediate declarative recall performance prior to sleep in sleep-restricted individuals, suggesting that the amount of learning achieved before sleep modulated the interaction between sleep spindles and hippocampal responses (Bergmann et al., 2012). However, no relationship was observed between hippocampal activity during spindles and overnight changes in memory recall in that study. Here we found a trend for a relationship with pre-to post-sleep changes in memory recall, in accordance with the hypothesis that neural processes in the hippocampus during fast spindles might modulate the consolidation of declarative information. Several differences between our study and that previous one should be noted in 

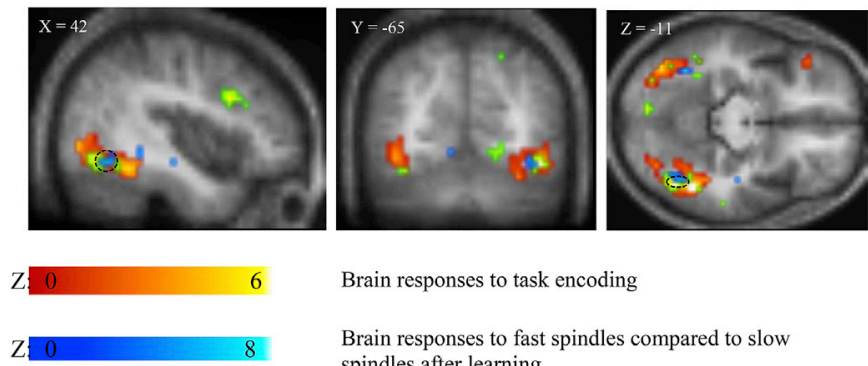

Brain responses to task encoding

Brain responses to fast spindles compared to slow spindles after learning

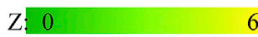

Brain responses to task recall, modulated by overnight change in recall performance

Fig. 5. Brain responses recruited by task encoding, fast spindles after encoding and task recall.

This map combines the brain responses to task encoding (in red, similar to Fig. 3A), the responses to fast spindles as compared to slow spindles (in blue, similar to Fig. 4A), and those to task recall after sleep compared to before sleep and modulated by the overnight change in recall performance (in green, similar to Fig. 3B), during the learning compared to the control night. Note the overlap between these three contrasts in the fusiform gyrus, as delineated by the black dotted circles. Displayed at $\mathrm{p}<0.02$ uncorrected, on the mean structural MRI. The color bars indicate the range of $\mathrm{Z}$ scores for each contrast.

that regard: sample sizes (14 subjects in our final sample, 9 in previous study), sleep pressure (participants were not sleep-restricted before learning in our study), tasks (previous study used a visuomotor task as control), and analyses (contrast between the occurrence of fast and slow spindles in our study to control for differences in baseline activity between nights; responses related to fast spindle amplitude without contrasting between spindle subtypes in previous study).

In addition to hippocampal and neocortical regions, we also found a trend for increased responses in the thalamus during fast spindles of the learning night. The thalamus is a critical structure for the generation of sleep spindles, as spindles have been shown to arise from the interactions between reticular thalamic neurons and thalamo-cortical neurons, the latter entraining cortical pyramidal neurons in spindle oscillations (Steriade et al., 1993). Previous fMRI studies consistently reported an association between sleep spindles and thalamic activity (Schabus et al., 2007; Caporro et al., 2012). The present findings suggest that this thalamic activity could be further enhanced following the encoding of new declarative learning. Interestingly these changes in thalamic responses with fast spindles during the learning night were not reflected by corresponding enhancements in spindle density (Table 1). Our results might thus indicate an increase in spindle-generating processes after learning, possibly facilitating spindle-mediated cortical reorganization, but not of a magnitude to produce a consistent increase in the number of generated spindles.

There are some limitations to our study. In particular, a possible confound inherent in our design was a potential order effect as the overlearning control condition always followed the initial experimental learning session. However, adopting a cross-over design in which half of the participants would have overlearned a face-sequence set as a control condition before the learning session on a different face-sequence set, would have rendered the design to investigate interference learning rather than memory consolidation only. Indeed, an overlearned facesequence control prior to the learning session of interest would have resulted in a strong memory representation interfering with the subsequent learning of a new face set. Furthermore, in our study, the fact that we observed a response in the fusiform gyrus not only during task encoding and fast spindles but also during task recall after including the difference in recall performance between the learning and control nights as a covariate, argues against the possibility of a non-specific order effect. Such order effect would have been most likely insensitive to learning performances.

In summary, our results demonstrate increased brain responses in the

fusiform gyrus both during declarative learning as well as during fast spindles of the subsequent sleep period following learning. This finding provides evidence for a cortical reactivation during fast spindles, especially in regions involved in prior learning before sleep. Importantly, these regions were also activated during recall after sleep, suggesting that these patterns of cortical activation and reactivation promote the offline consolidation of memory traces. In addition, during fast spindles after learning, there was a trend for a larger response in the hippocampus in proportion to the overnight memory change, suggesting that spindlerelated hippocampal reactivation might facilitate the cortical consolidation and recall of declarative memories. These findings contribute to the increasingly large body of evidence linking sleep spindles to the stabilization and integration of declarative information into long-term memory.

\section{Acknowledgements}

This work was supported by the Belgian National Funds for Scientific Research (FRS-FNRS), the University and University Hospital of Liège (Belgium). T.T.D.V. was also supported by the Natural Sciences and Engineering Research Council of Canada (RGPIN 436006-2013), the Canadian Institutes of Health Research (MOP 142191, PJT 153115 and PJT 156125), the Fonds de Recherche du Québec - Santé; the Canada Foundation for Innovation and Concordia University. AJ was supported by a Perform centre master's scholarship. M.S. was supported by an Erwin Schrödinger fellowship of the Austrian Science Fund (FWF; J2470-B02). O.G was also supported by the European Union's Horizon 2020 Framework Programme for Research and Innovation under the Specific Grant Agreement No. 785907 (Human Brain Project SGA2). We thank all the participants for their participation in this study.

\section{Appendix A. Supplementary data}

Supplementary data to this article can be found online at https://doi. org/10.1016/j.neuroimage.2019.03.051.

\section{References}

Allen, P.J., Josephs, O., Turner, R., 2000. A method for removing imaging artifact from continuous EEG recorded during functional MRI. Neuroimage 12, 230-239.

Andrade, K.C., Spoormaker, V.I., Dresler, M., Wehrle, R., Holsboer, F., Samann, P.G., Czisch, M., 2011. Sleep spindles and hippocampal functional connectivity in human NREM sleep. J. Neurosci. : Off. J/ Soc. Neurosci. 31, 10331-10339.

Bergmann, T.O., Molle, M., Diedrichs, J., Born, J., Siebner, H.R., 2012. Sleep spindlerelated reactivation of category-specific cortical regions after learning face-scene associations. Neuroimage 59, 2733-2742.

Caporro, M., Haneef, Z., Yeh, H.J., Lenartowicz, A., Buttinelli, C., Parvizi, J., Stern, J.M., 2012. Functional MRI of sleep spindles and K-complexes. Clin. Neurophysiol. 123, 303-309.

Clark, V.P., Maisog, J.M., Haxby, J.V., 1998. fMRI study of face perception and memory using random stimulus sequences. J. Neurophysiol. 79, 3257-3265.

Dang-Vu, T.T., Bonjean, M., Schabus, M., Boly, M., Darsaud, A., Desseilles, M., Degueldre, C., Balteau, E., Phillips, C., Luxen, A., Sejnowski, T.J., Maquet, P., 2011. Interplay between spontaneous and induced brain activity during human non-rapid eye movement sleep. Proc. Natl. Acad. Sci. U. S. A. 108, 15438-15443.

Dang-Vu, T.T., Schabus, M., Desseilles, M., Albouy, G., Boly, M., Darsaud, A., Gais, S., Rauchs, G., Sterpenich, V., Vandewalle, G., Carrier, J., Moonen, G., Balteau, E., Degueldre, C., Luxen, A., Phillips, C., Maquet, P., 2008. Spontaneous neural activity during human slow wave sleep. Proc. Natl. Acad. Sci. U. S. A. 105, 15160-15165.

De Gennaro, L., Ferrara, M., 2003. Sleep spindles: an overview. Sleep Med. Rev. 7, 423-440.

Diekelmann, S., Born, J., 2010. The memory function of sleep. Nat. Rev. Neurosci. 11, 114-126.

Eschenko, O., Molle, M., Born, J., Sara, S.J., 2006. Elevated sleep spindle density after learning or after retrieval in rats. J. Neurosci. 26, 12914-12920.

Fogel, S., Albouy, G., King, B.R., Lungu, O., Vien, C., Bore, A., Pinsard, B., Benali, H., Carrier, J., Doyon, J., 2017. Reactivation or transformation? Motor memory consolidation associated with cerebral activation time-locked to sleep spindles. PLoS One 12, e0174755.

Fogel, S.M., Smith, C.T., 2011. The function of the sleep spindle: a physiological index of intelligence and a mechanism for sleep-dependent memory consolidation. Neurosci. Biobehav. Rev. 35, 1154-1165.

Friston, K.J., Fletcher, P., Josephs, O., Holmes, A., Rugg, M.D., Turner, R., 1998. Eventrelated fMRI: characterizing differential responses. Neuroimage 7, 30-40. 
Gais, S., Molle, M., Helms, K., Born, J., 2002. Learning-dependent increases in sleep spindle density. J. Neurosci. 22, 6830-6834.

Grill-Spector, K., Knouf, N., Kanwisher, N., 2004. The fusiform face area subserves face perception, not generic within-category identification. Nat. Neurosci. 7, 555-562.

Iber, C., Ancoli-Israel, S., Chesson, A.L., Quan, S.F., 2007. The AASM Manual for the Scoring of Sleep and Associated Events. American Academy of Sleep Medicine, Westchester.

Kanwisher, N., McDermott, J., Chun, M.M., 1997. The fusiform face area: a module in human extrastriate cortex specialized for face perception. J. Neurosci. 17, 4302-4311.

Ketz, N., Jones, A.P., Bryant, N.B., Clark, V.P., Pilly, P.K., 2018. Closed-loop slow-wave tACS improves sleep-dependent long-term memory generalization by modulating endogenous oscillations. J. Neurosci. 38, 7314-7326.

Koo, P.C., Molle, M., Marshall, L., 2018. Efficacy of slow oscillatory-transcranial direct current stimulation on EEG and memory - contribution of an inter-individual factor. Eur. J. Neurosci. 47, 812-823.

Kumaran, D., Maguire, E.A., 2006. The dynamics of hippocampal activation during encoding of overlapping sequences. Neuron 49, 617-629.

Leclercq, Y., Balteau, E., Dang-Vu, T., Schabus, M., Luxen, A., Maquet, P., Phillips, C., 2009. Rejection of pulse related artefact (PRA) from continuous electroencephalographic (EEG) time series recorded during functional magnetic resonance imaging (fMRI) using constraint independent component analysis (cICA). Neuroimage 44, 679-691.

Leclercq, Y., Schrouff, J., Noirhomme, Q., Maquet, P., Phillips, C., 2011. fMRI artefact rejection and sleep scoring toolbox. Comput. Intell. Neurosci. 2011, 598206.

Lustenberger, C., Boyle, M.R., Alagapan, S., Mellin, J.M., Vaughn, B.V., Frohlich, F., 2016. Feedback-controlled transcranial alternating current stimulation reveals a functional role of sleep spindles in motor memory consolidation. Curr. Biol. 26, 2127-2136.

Luthi, A., 2014. Sleep spindles: where they come from, what they do. Neuroscientist 20, 243-256.

Marshall, L., Molle, M., Hallschmid, M., Born, J., 2004. Transcranial direct current stimulation during sleep improves declarative memory. J. Neurosci. 24, 9985-9992.

Molle, M., Bergmann, T.O., Marshall, L., Born, J., 2011. Fast and slow spindles during the sleep slow oscillation: disparate coalescence and engagement in memory processing. Sleep 34, 1411-1421.

Molle, M., Eschenko, O., Gais, S., Sara, S.J., Born, J., 2009. The influence of learning on sleep slow oscillations and associated spindles and ripples in humans and rats. Eur. J. Neurosci. 29, 1071-1081.
Molle, M., Marshall, L., Gais, S., Born, J., 2002. Grouping of spindle activity during slow oscillations in human non-rapid eye movement sleep. J. Neurosci. 22, 10941-10947.

Peigneux, P., Laureys, S., Fuchs, S., Collette, F., Perrin, F., Reggers, J., Phillips, C., Degueldre, C., Del Fiore, G., Aerts, J., Luxen, A., Maquet, P., 2004. Are spatial memories strengthened in the human hippocampus during slow wave sleep? Neuron 44, 535-545.

Pourtois, G., Schwartz, S., Seghier, M.L., Lazeyras, F., Vuilleumier, P., 2005. Viewindependent coding of face identity in frontal and temporal cortices is modulated by familiarity: an event-related fMRI study. Neuroimage 24, 1214-1224.

Rasch, B., Born, J., 2013. About sleep's role in memory. Physiol. Rev. 93, 681-766.

Rasch, B., Buchel, C., Gais, S., Born, J., 2007. Odor cues during slow-wave sleep prompt declarative memory consolidation. Science 315, 1426-1429.

Schabus, M., Dang-Vu, T.T., Albouy, G., Balteau, E., Boly, M., Carrier, J., Darsaud, A., Degueldre, C., Desseilles, M., Gais, S., Phillips, C., Rauchs, G., Schnakers, C., Sterpenich, V., Vandewalle, G., Luxen, A., Maquet, P., 2007. Hemodynamic cerebral correlates of sleep spindles during human non-rapid eye movement sleep. Proc. Natl. Acad. Sci. U. S. A. 104, 13164-13169.

Schabus, M., Gruber, G., Parapatics, S., Sauter, C., Klosch, G., Anderer, P., Klimesch, W., Saletu, B., Zeitlhofer, J., 2004. Sleep spindles and their significance for declarative memory consolidation. Sleep 27, 1479-1485.

Schabus, M., Hodlmoser, K., Gruber, G., Sauter, C., Anderer, P., Klosch, G., Parapatics, S., Saletu, B., Klimesch, W., Zeitlhofer, J., 2006. Sleep spindle-related activity in the human EEG and its relation to general cognitive and learning abilities. Eur. J. Neurosci. 23, 1738-1746.

Sirota, A., Csicsvari, J., Buhl, D., Buzsaki, G., 2003. Communication between neocortex and hippocampus during sleep in rodents. Proc. Natl. Acad. Sci. U. S. A. 100, 2065-2069.

Steriade, M., McCormick, D.A., Sejnowski, T.J., 1993. Thalamocortical oscillations in the sleeping and aroused brain. Science 262, 679-685.

Stickgold, R., 2013. Parsing the role of sleep in memory processing. Curr. Opin. Neurobiol. 23, 847-853.

Vuilleumier, P., Armony, J.L., Driver, J., Dolan, R.J., 2001. Effects of attention and emotion on face processing in the human brain: an event-related fMRI study. Neuron 30, 829-841.

Wislowska, M., Heib, D.P., Griessenberger, H., Hoedlmoser, K., Schabus, M., 2016. Individual baseline memory performance and its significance for sleep-dependent memory consolidation. Sleep Spindles Cortical Up States 1, 2-13. 